\title{
Method of Centre Manifold from Bifurcation Theory
}

\section{Dr. Basher Suleiman Othman}

Assistant Professor

Department of Mathematics College of Sciences \& Arts in Qilwah

Qilwah-Al-Baha University -Saudi Arabia

\begin{abstract}
The aim of this paper is to introduce tools from bifurcation theory is necessary in ways in our life particularly in the study of neural field equations set in the primary visual cortex. So we deal with saddlenode, trans- critical, pitchfork and Hopf. Bifurcations as an elementary bifurcation; directly related to the center manifold theory which is a canonical way to write differential equations.

We conclude this paper with an overview of bifurcations with symmetry by solving some problems and giving Branching Lemma as the equivariant result
\end{abstract}

Keywords: bifurcations theory- neural field- stable manifold- differential equations

\section{Elementary bifurcation}

Definition 1.1. In dynamical systems, a bifurcation occurs when a small smooth change made to the parameter values (the bifurcation parameters) of a system causes a sudden qualitative" or topological change in its behavior. Generally, at a bifurcation, the local stability properties of equilibrium, periodic orbits or other invariant sets changes.

\subsubsection{Bifurcation of dimension 1:}

Let us consider scalar differential equations of the form

$$
\frac{\mathrm{du}}{\mathrm{dt}}=\mathrm{f}(\mathrm{u}, \mu)
$$

Here the unknown $\mathrm{u}$ is a real-valued function of the time $\mathrm{t}$, and the vector field $\mathrm{f}$ is real- valued depending, besides $\mathrm{u}$, upon a parameter $\mu$. The parameter $\mu$ is the bifurcation parameter. We suppose that equation (1.1.1) is well-defined and satisfies the hypotheses of the Cauchy-Lipschitz theorem, such that for each initial condition there exists a unique solution of equation (1.1.1). Furthermore we assume that the vector field is of class $C^{\mathrm{k}}, \mathrm{k} \geq 2$, in a neighborhood of $(0,0)$ satisfying:

$$
\mathrm{f}(0,0)=0, \frac{\partial \mathrm{f}}{\partial \mathrm{u}}(0,0)=0
$$

The first condition shows that $u=0$ is an equilibrium of equation (1.1.1) at $\mu=0$. We are interested in local bifurcations that occur in the neighborhood of this equilibrium, when we vary the parameter $\mu$. The second condition is a necessary, but not sufficient, condition for the appearance of local bifurcations at $\mu=0$.

Remark 1.1. Suppose that the second condition is not satisfied:

$\partial \mathrm{f} / \partial \mathrm{u}(0,0) \neq 0$.

A direct application of the implicit function theorem shows that the equation $f(u, \mu)=0$

possesses a unique solution $\mathrm{u}=\mathrm{u}(\mu)$ in a neighborhood of 0 , for small enough $\mu$. In particular $\mathrm{u}=0$ is the only equilibrium of equation (1.1.1) in a neighborhood of 0 when $\mu=0$, and the same property holds for $\mu$ small enough. Furthermore, the dynamics of (1.1.1) in a neighborhood of 0 is qualitatively the same for all sufficiently small values of the parameter $\mu$, no bifurcation occurs for small values of $\mu$.

\subsubsection{Saddle-node bifurcation}


This is a fixed point which is an attractor from both sides in one pair of directions and an expeller on both sides of the other. Here is the phase diagram for a saddle point in a two-dimensional system. The 'stable manifold' is simultaneously the watershed of expeller and also the only line of attraction for the fixed point.

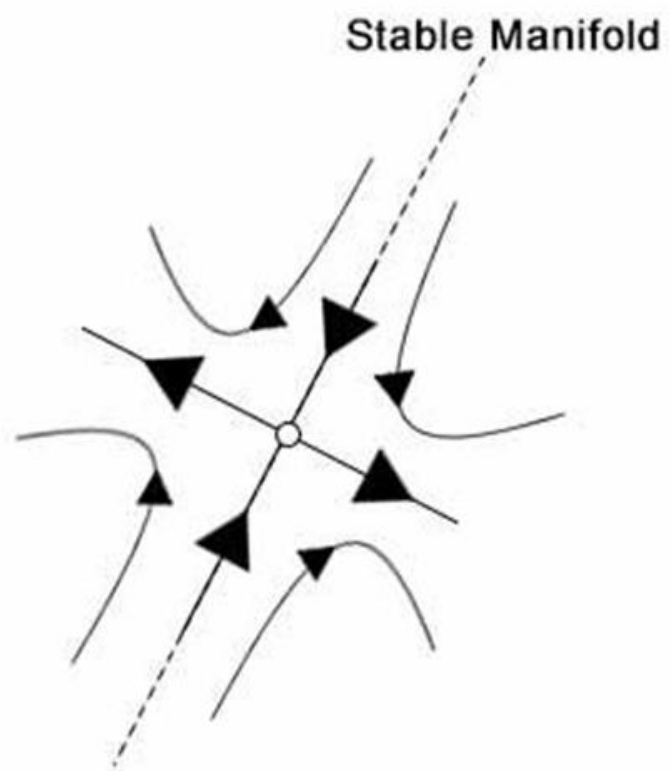

And let we state the following theorem which declares the fixed point in figure. For $f(u, \mu)=a \mu+b u^{2}+$ $o\left(|\mu|+u^{2}\right)$ as $(u, \mu) \rightarrow(0,0)$

Theorem 1.1 (Saddle-node bifurcation). Assume that the vector field $\mathrm{f}$ is of class $\mathrm{C}^{\mathrm{k}}, \mathrm{k} \geq 2$, in a neighborhood of $(0,0)$ and satisfies.

$$
\begin{aligned}
& \frac{\partial \mathrm{f}}{\partial \mu}(0,0)=0, \mathrm{a} \neq 0 \\
& \frac{\partial^{2} f}{\partial \mu^{2}}(0,0)=0,2 b \neq 0
\end{aligned}
$$

The following properties hold in neighborhood of 0 in $\mathrm{R}$ for small enough $\mu$.

(i) if $\mathrm{ab}<0$ (resp. $a b>0$ ) the differential equation has no equilibrium for $\mu<0$ (resp.for $\mu>0$ ),

(ii) if $a b<0$ (resp. $a b>0$ ) the differential equation possesses two equilibriumsu $\pm(\in), \in=\sqrt{|\mu|}$ for $\mu>$ 0 (resp. $\mu<0)$, with opposite stabilities. Furthermore, the map $\in \rightarrow \mathrm{u} \pm(\epsilon)$ is of class $\mathrm{C}^{\mathrm{k}-2}$ in a neighborhood of 0 in $\mathrm{R}$, and $\mathrm{u} \pm(\epsilon)=\mathrm{O}(\epsilon)$.

Then for equation (1.1.1), a saddle-node bifurcation occurs at $\mu=0$.

A direct consequence of conditions (1.1.3) is that $f$ has the expansion:

$$
f(u, \mu)=a \mu+b^{2}+o\left(|\mu|+u^{2}\right) \text { as }(u, \mu) \rightarrow(0,0)
$$

Application: 1.1. Consider the truncated equation

$$
\frac{\mathrm{du}}{\mathrm{dt}}=\mathrm{a} \mu+\mathrm{bu}^{2}
$$

Plot bifurcation diagrams in the $(u, \mu)$-plane of this truncated equation for different values of $a$ and $b$.

Proof. Since $a \neq 0$, we apply the implicit function theorem which implies the existence of unique solution $\mu$ $=g(u)$ for $u$ close to 0 of the equation $f(u, \mu)=0$, where $g$ is of class $C^{k}, k \geq 2$ in a neighborhood of the origin with $g(0)=0$. Its Taylor expansion is given by

$$
\mu=-\frac{b}{a} u^{2}+o\left(u^{2}\right)
$$

Consequently, if $a b \mu>0$ equation (1.1.1) has no equilibriums, one equilibrium $u=0$ if $\mu=0$ and a pair of equilibria

Finally, in the case $a b \mu<0$, we have:

$$
u \pm(\mu)= \pm \sqrt{-a \mu / b}+o(\sqrt{|\mu|}) \text { if } a b \mu<0
$$

$$
\frac{\partial \mathrm{f}}{\partial \mathrm{u}}(\mathrm{u} \pm(\mu), \mu)=2 \mathrm{bu} \pm \mathrm{o}(\sqrt{|\mu|})
$$

then the equilibrium $u-(\mu)$ is attractive, asymptotically stable when 
$\mathrm{b}>0$ and repelling, unstable when $\mathrm{b}<0$, where's, the equilibrium $\mathrm{u}+(\mu)$ has opposite stability properties.

\subsubsection{Pitchfork bifurcation}

Theorem 1.2 (Pitchfork bifurcation). Assume that the vector field $\mathrm{f}$ is of class $\mathrm{C}^{\mathrm{k}}, \mathrm{k} \geq 3$, in a neighborhood of $(0,0)$, that it is satisfies conditions (1.1.2), and that it is odd with respect to $u$ :

Furthermore assume that:

$$
f(-u, \mu)=-f(u, \mu)
$$

$$
\frac{\partial^{2} \mathrm{f}}{\partial \mu \partial \mathrm{u}}(0,0)=0, \mathrm{a} \neq 0, \frac{\partial^{3} \mathrm{f}}{\partial \mathrm{u}^{3}}(0,0)=0,6 \mathrm{~b} \neq 0
$$

The following properties hold in neighborhood of 0 in $\mathrm{R}$ for small enough

(i) if $a b<0$ (resp. $a b>0)$ the differential equation has one trivial equilibrium $u=0$

for $\mu<0$ (resp. for $\mu>0$ ). This equilibrium is stable when $b<0$ and unstable when $b>0$.

(ii) if $a b<0$ (resp. $a b>0$ ) the differential equation possesses the trivial equilibrium $u=0$ and two nontrivial equilibriums

$$
\mathrm{u} \pm(\epsilon), \in=\sqrt{|\mu|} \text { for } \mu>0(\text { resp. } \mu<0)
$$

which are symmetric, $\mathrm{u}+(\epsilon)=-\mathrm{u}-(\epsilon)$. The map

$$
\in \rightarrow \mathrm{u} \pm(\epsilon) \text { is of class } \mathrm{C}^{\mathrm{k}-3}
$$

In a neighborhood of 0 in $R$, and $u \pm(\epsilon)=O(\epsilon)$. The nontrivial equilibriums are stable when $b<0$ and unstable when $\mathrm{b}>0$, whereas the trivial equilibrium has opposite stability .Then for equation (1.1.1), a pitchfork bifurcation occurs at $\mu=0$.

\section{Center Manifold Theory:}

Theorem (Local Center Manifold Theorem)Let $f \in C^{r}(E)$, where $E$ is an open subset of $R^{n}$ containing the origin and $r \geq 1$. Suppose that $f(0)=0$ and that $\operatorname{Df}(0)$ has $c$ eigenvalues with zero real part, and $s=n-c$ eigenvalues with negative real part. The system (1) then can be written in diagonal form

$$
\dot{\mathrm{x}}=\mathrm{Cx}+\mathrm{F}(\mathrm{x}, \mathrm{y})
$$

$\dot{\mathrm{y}}=\mathrm{Py}+\mathrm{G}(\mathrm{x}, \mathrm{y})$

where $(x, y) \in R^{c} \times R^{s}, C$ is a square matrix with $c$ eigenvalues with zero real parts, $P$ is a square matrix with $s$ eigenvalues with negative real parts, and $\mathrm{F}(0)=\mathrm{G}(0)=0, \mathrm{DF}(0)=\mathrm{DG}(0)=0$; furthermore, there exists a $\delta>0$ and a function $\mathrm{h} \in \mathrm{C}^{\mathrm{r}}\left(\mathrm{N} \delta_{\delta}(0)\right), \mathrm{h}(0)=0, \mathrm{Dh}(0)=0$ the defines the local center manifold $\mathrm{W}^{\mathrm{c}}(0):=\left\{(\mathrm{x}, \mathrm{y}) \in \mathrm{R}^{\mathrm{c}} \times \mathrm{R}^{\mathrm{s}} \mid \mathrm{y}=\mathrm{h}(\mathrm{x})\right.$ for $\left.|\mathrm{x}|<\delta\right\}$ and satisfies

$\operatorname{Dh}(\mathrm{x})[\mathrm{Cx}+\mathrm{F}(\mathrm{x}, \mathrm{h}(\mathrm{x}))]=\mathrm{Ph}(\mathrm{x})+\mathrm{G}(\mathrm{x}, \mathrm{h}(\mathrm{x}))$

for $|\mathrm{x}|<\delta$, and the flow on the center manifold $\mathrm{W}^{\mathrm{c}}(0)$ is defined by the system of differential equations $\dot{\mathrm{x}}=\mathrm{Cx}+\mathrm{F}(\mathrm{x}, \mathrm{h}(\mathrm{x}))$

for all $\mathrm{x} \in \mathrm{R}^{\mathrm{c}}$ with $|\mathrm{x}|<\delta$.

This theorem can be used to determine the flow near non-hyperbolic equilibrium points. The strategy is:

1. Convert (1) in diagonal form (4)

2. Use a series expansion for the components of $\mathrm{h}(\mathrm{x})$ (up to the degree of accuracy we need, provided that $r$ is sufficiently large)

3. Determine the components of the expansion of $h(x)$ using (5)

4. Substitute this approximate expression of $h(x)$ into (6) to determine the flow.

\section{$\underline{\text { Applications }}$}

(1) From the above theorem to determine the qualitative behavior of the origin for the system

$$
\begin{gathered}
\dot{x}=x y \\
\dot{y}=-y-x^{2}
\end{gathered}
$$


Let

$$
\begin{gathered}
\mathrm{h}(\mathrm{x})=\mathrm{ax}^{2}+\mathrm{bx}^{3}+\cdots \\
\operatorname{Dh}(\mathrm{x})=2 \mathrm{ax}+3 \mathrm{bx}^{2}+\cdots \\
\operatorname{Dh}(\mathrm{x})[\mathrm{Cx}+\mathrm{F}(\mathrm{x}, \mathrm{h}(\mathrm{x}))]=\left(2 \mathrm{ax}+3 \mathrm{bx}^{2}+\cdots\right) \mathrm{x}\left(\mathrm{ax}^{2}+\mathrm{bx}^{3}+\cdots\right)
\end{gathered}
$$


$\downarrow \downarrow$ Collecting terms (Using(5))

$$
\mathrm{Ph}(\mathrm{x})+\mathrm{G}(\mathrm{x}, \mathrm{h}(\mathrm{x}))=-\left(a \mathrm{x}^{2}+\mathrm{bx}^{3}+\cdots\right)-\mathrm{x}^{2}
$$

$$
\begin{gathered}
O\left(x^{2}\right):-a-1=0 \\
O\left(x^{3}\right): b=0
\end{gathered}
$$

So $h(x)=-x^{2}+O\left(x^{4}\right)$. The flow on the center manifold is given by (6)

$\dot{\mathrm{x}}=\mathrm{F}(\mathrm{x}, \mathrm{h}(\mathrm{x}))=-\mathrm{x}^{3}+\mathrm{O}\left(\mathrm{x}^{5}\right)$

i.e., the system is stable.

(2): From the theorem of central manifold to determine the qualitative behavior of the origin for the system

$$
\begin{gathered}
\dot{\mathrm{x}}_{1}=-\mathrm{x}_{2}+\mathrm{x}_{1} \mathrm{y} \\
\dot{\mathrm{x}}_{2}=\mathrm{x}_{1}+\mathrm{x}_{2} \mathrm{y} \\
\dot{\mathrm{y}}=-\mathrm{y}-\mathrm{x}_{1}^{2}-\mathrm{x}_{2}^{2}+\mathrm{y}^{2}
\end{gathered}
$$

Solution(2): The system is already in the desired form, with

$$
\begin{aligned}
& \mathrm{C}=\left[\begin{array}{cc}
0 & -1 \\
1 & 0
\end{array}\right], \mathrm{P}=-1 \\
& \mathrm{~F}(\mathrm{x}, \mathrm{y})=\left[\begin{array}{l}
\mathrm{x}_{1} \mathrm{y} \\
\mathrm{x}_{2} \mathrm{y}
\end{array}\right] \text { and } \mathrm{G}(\mathrm{x}, \mathrm{y})=-\mathrm{x}_{1}^{2}-\mathrm{x}_{2}^{2}+\mathrm{y}^{2} \text {. Let } \\
& \mathrm{h}\left(\mathrm{x}_{1}, \mathrm{x}_{2}\right)=\mathrm{ax}_{1}^{2}+\mathrm{bx}_{1} \mathrm{x}_{2}+\mathrm{cx}_{2}^{2}+\cdots \\
& \operatorname{Dh}(\mathrm{x})=\left[2 \mathrm{ax}_{1}+\mathrm{bx}_{2}+\cdots, \quad \mathrm{bx}_{1}+2 \mathrm{cx}_{2} \cdots\right]
\end{aligned}
$$

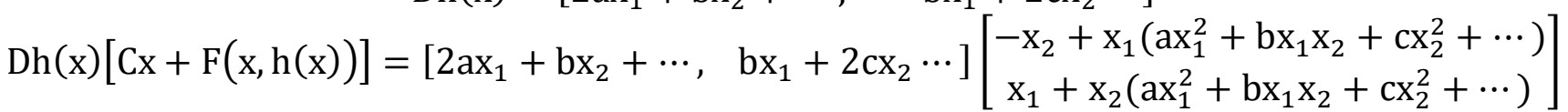

$$
\begin{aligned}
& \mathrm{Ph}(\mathrm{x})+\mathrm{G}(\mathrm{x}, \mathrm{h}(\mathrm{x}))=-\left(2 \mathrm{ax}_{1}^{2}+\mathrm{bx}_{1} \mathrm{x}_{2}+\mathrm{cx}_{2}^{2}+\cdots\right)-\mathrm{x}_{1}^{2}-\mathrm{x}_{2}^{2}+\left(\mathrm{ax}_{1}^{2}+\mathrm{bx}_{1} \mathrm{x}_{2}+\mathrm{cx}_{2}^{2}+\cdots\right)^{2}
\end{aligned}
$$

$\downarrow \downarrow$ Collecting terms (Using(5))

$$
\begin{gathered}
x_{1}^{2}: b=-a-1 \\
x_{2}^{2}:-b=-c-1 \\
x_{1} x_{2}:-2 a+2 c=-b
\end{gathered}
$$

we get $a=-1, b=0, c=-1$, and so $h\left(x_{1}, x_{2}\right)=-x_{1}^{2}-x_{2}^{2}+O\left(|x|^{3}\right)$. The flow on the center manifold is given by (6)

$$
\begin{gathered}
\dot{\mathrm{x}}_{1}=-\mathrm{x}_{2}+\mathrm{x}_{1}\left(-\mathrm{x}_{1}^{2}-\mathrm{x}_{2}^{2}+\mathrm{O}\left(|\mathrm{x}|^{3}\right)\right)=-\mathrm{x}_{2}-\mathrm{x}_{1}^{3}-\mathrm{x}_{1} \mathrm{x}_{2}^{2}+\mathrm{O}\left(|\mathrm{x}|^{4}\right) \\
\dot{\mathrm{x}}_{2}=\mathrm{x}_{1}+\mathrm{x}_{2}\left(-\mathrm{x}_{1}^{2}-\mathrm{x}_{2}^{2}+\mathrm{O}\left(|\mathrm{x}|^{3}\right)\right)=\mathrm{x}_{1}-\mathrm{x}_{2}^{3}-\mathrm{x}_{1}^{2} \mathrm{x}_{2}+\mathrm{O}\left(|\mathrm{x}|^{4}\right)
\end{gathered}
$$

\section{Results:}

The center manifold theorem allows us to determine the local behavior of the system by looking at the flow on a lower dimensional manifold, i.e., instead of working with an n-dimensional system, we can just deal with a c-dimensional one (i.e., we "reduce" the full n-dimensional system into a c-dimensional system). In this example, instead of trying to determine the behavior of the full 3-d system, we reduced the problem to determine the behavior of the 2-d system above.

To determine the stability of the origin, let's try changing to polar coordinates

$$
\begin{gathered}
\dot{r} \dot{r}=x_{1} \dot{x}_{1}+x_{2} \dot{x}_{2} \\
=x_{1}\left(-x_{2}-x_{1}^{3}-x_{1} x_{2}^{2}\right)+x_{2}\left(x_{1}-x_{2}^{3}-x_{1}^{2} x_{2}\right)+O\left(|r|^{5}\right) \\
=-x_{1}^{4}-2 x_{1}^{2} x_{2}^{2}-x_{2}^{4}+O\left(|x|^{5}\right)=-\left(x_{1}^{2}+x_{2}^{2}\right)^{2}+O\left(|r|^{5}\right) \\
=-r^{4}+O\left(|r|^{5}\right) \\
\dot{r}=-r^{3}+O\left(|x|^{4}\right)
\end{gathered}
$$

i.e., the system is stable.

Applications:

(3) Determine a center manifold for the rest point at the origin of the system

$$
\dot{\mathrm{x}}=-\mathrm{xy}
$$




$$
\dot{y}=-y+x^{2}-2 y^{2}
$$

a) Determine a center manifold for the rest point at the origin of the system and a differential equation for the dynamics on this center manifold.

b) Show that every solution of the system is attracted to the center manifold.

c) Determine the stability type of the rest point at the origin.

Solution(3)

Progress so far: By the Invariant manifold theorem (IMT) we look for an invariant manifold as a graph of a function of the form,

$$
y=h(x)=a_{0}+a_{1} x+\alpha x^{2}+\beta x^{3}+\gamma x^{4}+\delta x^{5}+\theta x^{6}+O\left(x^{7}\right)
$$

By IMT we get that the constant term and the $\mathrm{x}$ term to be zero. By invariance we need to have thaty $=$ $\mathrm{h}^{\prime}(\mathrm{x}) \dot{\mathrm{x}}$. After some tedious computations and comparing coefficients of $\mathrm{x}$ of both sides we get,

$$
\mathrm{h}(\mathrm{x})=\mathrm{x}^{2}-4 \mathrm{x}^{4}+16 \mathrm{x}^{6}-64 \mathrm{x}^{8}+\cdots
$$

Thus we can conclude that (using properties of geometric series)

$$
h(x)=\frac{x^{2}}{1+2 x^{2}}
$$

for $\mathrm{x}$ near zero. This is the required center manifold. A differential equation for the dynamics of the center manifold is $\dot{\mathrm{x}}=-\mathrm{xh}(\mathrm{x})$ with $\mathrm{h}(\mathrm{x})$ as above.

We answer part (c)

$$
\dot{x}=-x\left(x^{2}-4 x^{4}+16 x^{6}-64 x^{8}+\cdots\right)=-x^{3}+0\left(x^{5}\right)
$$

and since the coefficient of $x^{3}$ is negative it follows that the origin is an asymptotically stable rest point for the center manifold and hence for the original system.

(4): As the above theorem to determine the qualitative behavior of the origin for the system

$$
\begin{gathered}
\dot{x}=a x^{2}+b x y+c y^{2} \\
\dot{y}=-y+d x^{2}+e x y+f y^{2}
\end{gathered}
$$

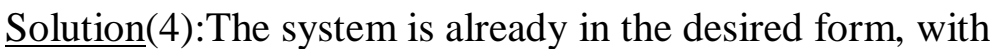

$\mathrm{C}=0, \mathrm{P}=-1, \mathrm{~F}(\mathrm{x}, \mathrm{y})=\mathrm{ax}^{2}+\mathrm{bxy}+\mathrm{cy}^{2}$ and

$\mathrm{G}(\mathrm{x}, \mathrm{y})=\mathrm{dx}^{2}+$ exy $+\mathrm{fy}^{2}$. Let

$$
\begin{gathered}
\mathrm{h}(\mathrm{x})=\mathrm{k}_{2} \mathrm{x}^{2}+\mathrm{k}_{3} \mathrm{x}^{3}+\cdots \\
\mathrm{Dh}(\mathrm{x})=2 \mathrm{k}_{2} \mathrm{x}+3 \mathrm{k}_{3} \mathrm{x}^{2}+\cdots
\end{gathered}
$$

$\mathrm{Dh}(\mathrm{x})[\mathrm{Cx}+\mathrm{F}(\mathrm{x}, \mathrm{h}(\mathrm{x}))]=\left(2 \mathrm{k}_{2} \mathrm{x}+3 \mathrm{k}_{3} \mathrm{x}^{2}+\cdots\right)\left(\mathrm{ax} \mathrm{x}^{2}+\mathrm{bx}\left(\mathrm{k}_{2} \mathrm{x}^{2}+\mathrm{k}_{3} \mathrm{x}^{3}+\cdots\right)+\mathrm{c}\left(\mathrm{k}_{2} \mathrm{x}^{2}+\mathrm{k}_{3} \mathrm{x}^{3}+\cdots\right)^{2}\right)$

$\mathrm{Ph}\left(\mathrm{x} 0+\mathrm{G}(\mathrm{x}, \mathrm{h}(\mathrm{x}))=-\left(\mathrm{k}_{2} \mathrm{x}^{2}+\mathrm{k}_{3} \mathrm{x}^{3}+\cdots\right)+\mathrm{dx} \mathrm{x}^{2}+\mathrm{ex}\left(\mathrm{k}_{2} \mathrm{x}^{2}+\mathrm{k}_{3} \mathrm{x}^{3}+\cdots\right)+\mathrm{f}\left(\mathrm{k}_{2} \mathrm{x}^{2}+\mathrm{k}_{3} \mathrm{x}^{3}+\cdots\right)^{2}\right.$

$\downarrow \downarrow$ Collecting terms (Using (5))

$$
\begin{gathered}
\mathrm{O}\left(\mathrm{x}^{2}\right):-\mathrm{k}_{2}+\mathrm{d}=0 \\
\mathrm{O}\left(\mathrm{x}^{3}\right): 2 \mathrm{k}_{2} \mathrm{a}=-\mathrm{k}_{3}+\mathrm{ek}_{2} \rightarrow \mathrm{k}_{3}=(\mathrm{e}-2 \mathrm{a}) \mathrm{k}_{2}
\end{gathered}
$$

So $h(x)=d x^{2}+(e-2 a) d x^{3}+O\left(x^{4}\right)$. The flow on the center manifold is given by (6)

$$
\begin{aligned}
& \dot{\mathrm{x}}=\mathrm{F}(\mathrm{x}, \mathrm{h}(\mathrm{x}))=\mathrm{ax}^{2}+\mathrm{bx}\left(\mathrm{dx^{2 }}+(\mathrm{e}-2 \mathrm{a}) \mathrm{dx}^{3}+\mathrm{O}\left(\mathrm{x}^{4}\right)\right)+\mathrm{c}\left(\mathrm{dx^{2 }}+(\mathrm{e}-2 \mathrm{a}) \mathrm{dx^{3 }}+\mathrm{O}\left(\mathrm{x}^{4}\right)\right)^{2} \\
& =a x^{2}+b d x^{3}+\left(b d(e-2 a)+c^{2}\right) x^{4}+0\left(x^{5}\right)
\end{aligned}
$$

So unstable fora $\neq 0$. For $a=0$ and $b d>0$ unstable, $b d<0$ stable. For $a=0$, $b d=0, c \neq 0$ it is unstable.

(5): From the above theorem to determine the qualitative behavior of the origin for the system

$$
\begin{gathered}
\dot{x}=-x^{3} \\
\dot{y}=-y+x^{2}
\end{gathered}
$$

Solution(5):First, it is clear that the system is stable since the flow on the center manifold is $\dot{\mathrm{x}}=-\mathrm{x}^{3}$.

Let's calculate the center manifold anyway. The system is already in the desired form, with $\mathrm{C}=0, \mathrm{P}=$ $-1, F(x, y)=-x^{3}$ and $G(x, y)=x^{2}$. Let

$$
\begin{gathered}
h(x)=a_{2} x^{2}+a_{3} x^{3}+\cdots \\
D h(x)=2 a_{2} x+3 a_{3} x^{2}+\cdots \\
\operatorname{Dh}(x)[C x+F(x, h(x))]=-\left(2 a_{2} x+3 a_{3} x^{2}+\cdots\right) x^{3}
\end{gathered}
$$


$\downarrow \downarrow$ Collecting terms (Using (5))

$$
P h(x)+G(x, h(x))=-\left(a_{2} x^{2}+a_{3} x^{3}+\cdots\right)+x^{2}
$$

$$
\begin{gathered}
O\left(x^{2}\right):-a_{2}+1=0 \\
O\left(x^{3}\right): a_{3}=0 \\
O\left(x^{4}\right):-2 a_{2}=-a_{4} \rightarrow a_{4}=2
\end{gathered}
$$

In general, $\mathrm{a}_{2 \mathrm{k}+1}=0$ and $\mathrm{a}_{\mathrm{n}+2}=\mathrm{na}_{\mathrm{n}}$ for $\mathrm{n}$ even. So the Taylor series $\mathrm{x}^{2}+2 \mathrm{x}^{4}+8 \mathrm{x}^{6}+\cdots$ which diverges for $\mathrm{x} \neq 0$. What happened? Let's try to solve for $\mathrm{h}(\mathrm{x})$ without using the power expansion

$$
\begin{gathered}
\frac{\mathrm{dh}(\mathrm{x})}{\mathrm{dx}}\left(-\mathrm{x}^{3}\right)=-\mathrm{h}(\mathrm{x})+\mathrm{x}^{2} \\
\downarrow \downarrow \\
\mathrm{h}^{\prime}=\frac{1}{\mathrm{x}^{3}} \mathrm{~h}-\frac{1}{\mathrm{x}}
\end{gathered}
$$

The existence and uniqueness theorem does not apply (since the vector field is not continuous in $\mathrm{x}$ at 0 ) . Indeed using mathematica to solve, it appears to give a continues of solutions (all satisfying $h(0)=0$, $\left.\mathrm{h}^{\prime}(0)=0\right)$,

where $\mathrm{a}$ is some constant and

$$
\mathrm{h}(\mathrm{x})=\exp \left(-\frac{1}{2 \mathrm{x}^{2}}\right) \mathrm{a}+\frac{1}{2} \exp \left(-\frac{1}{2 \mathrm{x}^{2}}\right) \text { ExpIntegral } \mathrm{Ei}\left(\frac{1}{2 \mathrm{x}^{2}}\right)
$$

$$
\text { ExpIntegral } \operatorname{Ei}(\mathrm{z})=\operatorname{Ei}(\mathrm{z})=-\int_{-\mathrm{z}}^{\infty} \frac{\mathrm{e}^{-\mathrm{t}}}{\mathrm{t}} \mathrm{dt}
$$

Remarks:

- There may be many functions $\mathrm{h}(\mathrm{x})$ that determine different center manifolds. However, the flows on

- the different center manifolds are determined by 6 and are all topologically equivalent near the origin.

- For an analytical system, if the series expansion of $\mathrm{h}$ converges, then there exists a unique analytical center manifold.

- For a analytical system (even polynomial), if the series does not converge, then an analytical center manifold need not exist.

Theorem : Let $f \in C^{1}(E)$, where $E$ is an open subset of $R^{n}$ containing the origin. Suppose that $f(0)=0$ and that $\operatorname{Df}(0)=\operatorname{diag}[\mathrm{C}, \mathrm{P}, \mathrm{Q}]$, where the square matric $\mathrm{C}$ has $\mathrm{c}$ eigenvalues with zero real part, the square matric $P$ has $S$ eigenvalues with negative real part, and the square matric $Q$ has $u=n-c-s$ eigenvalues with positive real part. Then there exist $\mathrm{C}^{1}$ functions $\mathrm{h}_{1}(\mathrm{x}), \mathrm{h}_{2}(\mathrm{x})$ satisfying

$$
\begin{aligned}
& D_{1}(x)\left[C x+F\left(x, h_{1}(x), h_{2}(x)\right)\right]=P h_{1}(x)+G\left(x, h_{1}(x), h_{2}(x)\right) \\
& D h_{2}(x)\left[C x+F\left(x, h_{1}(x), h_{2}(x)\right)\right]=Q h_{2}(x)+H\left(x, h_{1}(x), h_{2}(x)\right)
\end{aligned}
$$

in a neighborhood of the origin such that the nonlinear system (1) which can be written as

is topologically conjugate to the $\mathrm{C}^{1}$ system

$$
\begin{aligned}
& \dot{\mathrm{x}}=\mathrm{Cx}+\mathrm{F}(\mathrm{x}, \mathrm{y}, \mathrm{z}) \\
& \dot{\mathrm{y}}=\mathrm{Py}+\mathrm{G}(\mathrm{x}, \mathrm{y}, \mathrm{z}) \\
& \dot{\mathrm{z}}=\mathrm{Qz}+\mathrm{H}(\mathrm{x}, \mathrm{y}, \mathrm{z})
\end{aligned}
$$

$$
\begin{gathered}
\dot{\mathrm{x}}=\mathrm{Cx}+\mathrm{F}\left(\mathrm{x}, \mathrm{h}_{1}(\mathrm{x}), \mathrm{h}_{2}(\mathrm{x})\right) \\
\dot{\mathrm{y}}=\mathrm{Py} \\
\dot{\mathrm{z}}=\mathrm{Qz}
\end{gathered}
$$

for $(\mathrm{x}, \mathrm{y}, \mathrm{z}) \in \mathrm{R}^{\mathrm{c}} \times \mathrm{R}^{\mathrm{s}} \times \mathrm{R}^{\mathrm{u}}$ in a neighborhood of the origin.

\section{References}

[1] H. K. Khalil. Nonlinear Systems. Prentice Hall, 3rd edition, 2002.

[2] L. Perko. Differential Equations and Dynamical Systems. Springer, 3rd edition, 2001. 
[3] S. H. Strogatz. Nonlinear Dynamics and Chaos: With Applications to Physics, Biology, Chemistry, and Engineering. Westview Press, 2001. 